\title{
C-I
}

\section{The Electronic Structure of Heavy Element Complexes}

Introduction

This is a proposal for the renewal of the PI's grant from the Heavy Element Chemistry Program of the Division of Chemical Sciences of the Office of Energy Research of the U. S. Department of Energy. We are gratified with the progress we have made during the current period of this grant, which has been an exciting period of transition with respect to the methodology used in this research and the types of systems that were investigated. During this period, we have made considerable advancements in the understanding of the bonding in heavy element complexes, and in the application of more sophisticated electronic structure theories.

We view our role in research into the electronic structure of heavy element compounds to be primarily on the applications end of the spectrum. Our group has strengths in inorganic chemistry, electronic structure theory, and applications of supercomputing, and we believe that our most important function in the heavy element community is to provide theoretical results and explanations that are significant to experimental chemists in the field. Therefore, we have put our highest priority on obtaining experimentally relevant theoretical results for the heavy element community. In so doing, we have relied on the excellent theoretical groups at U.S. National Laboratories and elsewhere who are making significant advances to the methodologies and codes for heavy element electronic structure theory. We have strong and productive interactions with many of these theory groups, and will maintain those in the future.

The next three year period promises to be a very active time for our research in heavy element electronic structure theory for several reasons: (1) Advances in electronic structure methodologies, especially with respect to density functional theory (DFT), affords us the opportunity to perform much more accurate relativistic calculations, including, for example, relativistic geometry optimizations and subsequent vibrational frequency calculations as well as first-principle calculations of NMR, ESR, UVvis spectra, solvent effects, and redox properties. (2) Advances in massively parallelized ab initio and DFT codes enable us for the first time to address chemically interested problems using real chemical ligands such as $\mathrm{Me}, \mathrm{Et}, \mathrm{Bu}, \mathrm{Ph}, \mathrm{Cp}$, and $\mathrm{Cp}^{*}$, which had to be simulated previously by pseudoatoms/groups. (3) Advances in the resources available to us at the Ohio Supercomputer Center (OSC) and at National Laboratories will enable us to explore heavy-element systems that were previously not amenable to theoretical study because of size limitations. (4) Our work continues to attract the attention of actinide theorists and experimentalists, particularly at National Laboratories. During the present grant period, we have continued our strong link with researchers at Los Alamos National Laboratory (LANL), and have also injtiated collaborative efforts with researchers at Argonne National Laboratory (ANL), Lawrence Livermore National Laboratory (LLNL), and Pacific Northwest National Laboratory (PNNL).

This proposal will begin with a report on the progress made during the previous grant period. This will be followed by a description of the scientific activities that will be undertaken during the 200003 grant period.

Progress to Date

Technological Advances and Current Methodologies

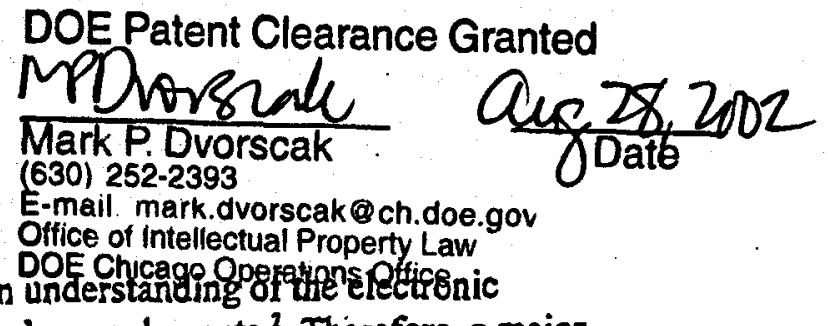

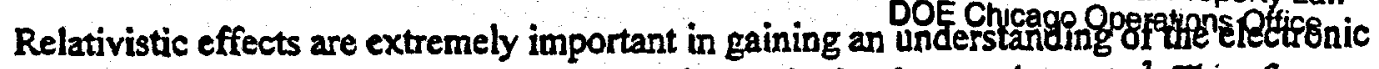
struoture of compounds of the actinides, transactinides, and other heavy elements. ${ }^{2}$ Therefore, a major part of our continual benchmarking has been the proper inclusion of the appropriate relativistic effects 


\section{DISCLAIMER}

This report was prepared as an account of work sponsored by an agency of the United States Government. Neither the United States Government nor any agency thereof, nor any of their employees, makes any warranty, express or implied, or assumes any legal liability or responsibility for the accuracy, completeness, or usefulness of any information, apparatus, product, or process disclosed, or represents that its use would not infringe privately owned rights. Reference berein to any spe. cific commercial product, process, or service by trade name, trademark, manufacturer, or otherwise does not necessarily constitute or imply its endorsement, recommendation, or favoring by the United States Government or any agency thereof. The views and opinions of authors expressed herein do not necessarily state or reflect those of the United States Governmeat or any agency thereof. 


\section{DISCLAIMER}

Portions of this document may be illegible in electronic image products. Images are produced from the best available original document. 
for the properties under study.

In traditional ab initio formalisms, the fundamental one-electron operator of relativistic quantum mechanics is the Dirac operator. ${ }^{3}$ An analogous many-body Hamiltonian is not known, and a proper quantum-electrodynamical treatment of corresponding many-body effects is beyond the scope of traditional quantum chemical techniques. For this reason, we typically use an approximate construction such as the Dirac-Fock operator as our starting point in relativistic electronic structure calculations. Under this formalism, the one-electron part of the nonrelativistic Fock operator is replaced by the independent particle Dirac equation, which includes the electronic kinetic energy and nuclear attraction operators. The two-particle electron-electron interaction is handled as in nonrelativistic calculations and the molecular wavefunction is represented as an antisymmetrized product of the one-electron Dirac-Fock orbitals. In this way, the relativistic mass-velocity and Darwin effects, which are the so-called scalor relativistic effects, are handled implicitly. The other major relativistic effect on electronic structure is the coupling of electronic spin and orbital angular momenta. Spin-orbit coupling can be included as a perturbation on scalar relativistic effects, or can be directly calculated by adding a spin-orbit operator to the Dirac-Fock operator.

In the DFT formalism, the inclusion of scalar relativistic effects and spin-orbit coupling effects are straightforward because the Kohn-Sham equation is formally similar to the Hartree-Fock equation except that the exact exchange-energy operator is replaced by the exchange-correlation functionals. As will be disoussed in greater detail below, we find that the use of scalar relativistic effects in the DFT formalism, without spin-orbit effects, generally leads to highly accurate calculated molecular geometries and vibrational frequencies for the ground-state properties of actinide complexes. The incorporation of scalar relativistic effects only in a single-configuration DFT method also leads to an easier description of the electronic structure inasmuch as one still has one-electron orbitals and the separation of spatial and spin wavefunctions. The inclusion of spin-orbit relativistic effects are, as expected, critical for the accurate calculation of optical transitions and excited-state properties of actinide-containing molecules. The description of the electronic structure of molecules with spin-orbit effects is far more complicated owing to the need for using double-group descriptions, but recent theoretical and programming developments allow us to discuss the spin-orbit effects on molecular properties in a way that is more understandable to scientists who are not entirely comfortable with double-group descriptions of molecular electronic structure.

Our choice of electronic structure methods for this project has evolved through the years. Our earliest calculations on actinide systems ${ }^{s}$ were carried out using the quasirelativistic X $\alpha$-SW molecular orbital method, which was one of the few methods at the time that allowed us to study large f-element systems with reasonable treatment of the scalar relativistic effects. Although the $\mathrm{X} \alpha-\mathrm{SW}$ method was an excellent starting point for us to explore the bonding in organoactinide complexes," the shortcomings in the model Hamiltonian used in the method led us to look for more accurate methodologies. To that end, we explored the relativistic implementation of the discrete variational X $\alpha$ (DV-X $\alpha$ ) method, which freed us from the "muffin-tin" approximation implicit to the X $\alpha$-SW method, and also allowed us to include spin-orbit relativistic effects in addition to scalar relativistic effects."

The $\mathrm{X} \alpha$ methods were, in essence, the first local density functional (LDF) theory methods applied to molecular systems. In relativistic LDF approaches, such as that implemented in the DV-Xa method, the Dirac-Fock two-electron terms are replaced with an exact density-based Coulomb repulsion operator and an approximate LDF exchange-correlation operator.' In the DV-X $\alpha$ method, a radial basis set for each atom is provided by numerical solution of the atomic Dirac-Fock equations and for a variety of neutral and cationic states of the species in question. The result of such an atomic calculations is a set 
of $j i$-coupled, two-component spinors. The molecular orbitals are then expanded as symmetry adapted linear combinations of these spinors, which span the additional representations of the molecular double point group. The molecular Dirac-Fock-Slater equations are then solved numerically to self-consistency over a three-dimensional grid of points. This technique has shown itself to be quite effective in providing a reasonably quantitative molecular orbital deseription of the species examined and in its ability to predict molecular ionization potentials and optical spectra. However, the reliance on numerical basis functions, and the resultant difficulties in three-dimensional numerical quadrature, substantially reduced our ability to calculate reliable total energies. We have found that molecular energies calculated in such a way are unreliable unless an untenably dense grid of integration points is used. This difficulty precluded our use of the DV-X $\alpha$ method for the optimization of molecular geometries. Thus, while relativistic LDF theory in the form of the DV-X $\alpha$ method remains a powerful tool in the description of molecular electronic structure, it was clear that we could not reasonably and getserally use this method for the calculation of molecular geometries in actinide systems.

In recent years, we and others have found that relativistic generalized-gradient (or non-local) density functional theory is an outstanding tool for the caloulation of the electronic structure of actinide complexes. ${ }^{10}$ In the generalized-gradient approach (GGA) implementation of DFT, additional terms are added to account for gradient corrections to the exchange-correlation functionals. There are a variety of generalized gradient approaches in DFT. In our experience, which includes extensive benchmarking of different gradient-corrected functionals, the best pure density-functionals for actinide compounds are the approaches of Becke and Perdew (BP86) ${ }^{11}$ and, especially, of Perdew and Wang (PW91). ${ }^{12}$ Most of the results presented here on actinide complexes, and most of our future work on actinide-containing systems, will involve the use of relativistic gradient-corrected DFT. We have tested extensively the correlations between the choice of functional, the degree of relativistic correction used, and the accuracy with which molecular properties are calculated. Significantly, we have determined that the caloulated geometries and vibrational frequencies of actinide complexes are minimally affected by the inclusion of spin-orbit effects. Thus, only scalar relativistic effects need be included in calculations of the geometries and frequencies of actinide complexes, which simplifies the calculations and the interpretation of them. We will be publishing some of these results as an invited book chapter in a forthcoming monograph. ${ }^{13}$

We have benefitted greatly from the development of suitable commercially available DFT codes that incorporate the functionals and features that we desire. In particular, we have found the Amsterdam Density Functional code (Theoretical Chemistry, Vrije Universiteit, Amsterdam, The Netherlands), developed by Baerends et al. $^{14}$ and incorporating the relativistic extensions first proposed by Snijders et al., ${ }^{15}$ to be a superbly developed and robust package for our needs. The combination of the computationally efficiency of the ADF code and the access to advanced supercomputers allows calculations to be carried out on very large actinide systems that are not amenable to study by traditional correlated $a b$ initio approaches. The recent efficient parallelization of this code has further enabled us to address even larger real chemical systems that are of great interest in the actinide community. In our view and experience, one of the big advantages of the ADF methodology is the use of Slater-type-orbital (STO) basis sets, which are generally more accurate than the Gaussian-type-orbital (GTO) basis sets used in most other ab initio and DFT codes. Further, the efficient implementation of the integration scheme in the ADF code allows us to use much larger basis sets, usually triple-zeta plus polarization or diffuse functions. The theoretical advances made by the Amsterdam group and others, such as the ability to calculate analytical energy gradients and analytical second-order energy derivatives for f-orbital basis sets, to calculate NMR and ESR parameters, to incorporate solvent effects, and to undertake timedependent DFT calculations, have made the ADF program one of the most comprehensive and efficient computational codes for dealing with a number of physical and chemical properties of heavy systems. These advances enable us for the first time to be able to address many of the very interesting chemical problems in actinide chemistry. We anticipate that a tremendous amount of really challenging actinide 
systems will be investigated in our next grant period.

Most of our investigations of the chemistry of small molecules of the transactinide elements have been carried out by using high-quality correlated $a b$ initio methods, such as the relativistic configuration interaction method of Pitzer et al. ${ }^{16}$ and the coupled-cluster approach of Bartlett et al. ${ }^{17}$ A key to the successful use of these methods was the derivation of suitable relativistic effective core potentials (RECPs) for the transactinide elements. To that end, we have collaborated with Professor W. C. Emler (Stevens Institute of Technology) to generate RECPs with spin-orbit operators for the later actinides and the transactinides through element $118 .^{18}$

\section{Specific Applications}

Organoactinide Sandwich Complexes. Sandwich complexes of the actinide elements, An( $\eta^{n}$ $\left.\mathrm{C}_{n} \mathrm{H}_{n}\right)_{2}$, have been central to the development of modern organoactinide chemistry. The remarkable discovery of uranocene, $\mathrm{U}(\mathrm{COT})_{2}\left(\mathrm{COT}=\eta^{2}-\mathrm{C}_{8} \mathrm{H}_{8}\right)$, by Streitwieser and Müller-Westerhoff in 1968 demonstrated that organoactinide complexes could adopt structural motifs that are unprecedented in organotransition metal chemistry. ${ }^{19}$ This chemistry has since expanded to include the synthesis of a wide variety of other actinocenes $\mathrm{An}(\mathrm{COT})_{2}(\mathrm{An}=\mathrm{Th}, \mathrm{Pa}, \mathrm{Np}, \mathrm{Pu}, \mathrm{Am})$ and actinocene anions. ${ }^{20}$ Several theoretical methods have been used to elucidate the electronic structures, bonding, and electronic spectra of various actinocenes and lanthanocenes..$^{21.22 .23 .24 .25}$

We addressed several aspects of organoactinide sandwich chemistry during the current grant period. First, we explored the electronic structure and optical transitions of protactinocene, $\mathrm{Pa}(\mathrm{COT})_{2}$. Protactinocene is a $\mathrm{Pa}(\mathrm{IV}) \mathrm{f}^{2}$ complex and as such should provide the most direct data about the influence of the (COT) ligand field on a sf electron. Indeed, at the end of his classic review paper on the ligand field theory of f-ortital sandwich complexes, Warren proposed that "high upon the list of desirable experimental information must however be a study of the magnetic and electronic spectral properties of the $5 \mathrm{f}^{1}$ complex, $\mathrm{Pa}$ (COT), ESR investigation of $\mathrm{f}^{1}$ and $\mathrm{f}^{\mathrm{f}}$ systems, measurement of anisotropic susceptibilities, and further spectral and MCD studies of all the actinocenes." 26 However, because of the small quantities of protactinium available and its pronounced radioactivity, the chemistry of protactinium compounds is still largely undeveloped, thus hampering a systematic comparison across the early actinide series. Although more than two decades have passed since Warren's review, the available data on protactinocene are scant; only $x$-ray powder diffraction data and some vibrational and visible spectra have been experimentally reported for protactinocene or its analog with $1,3,5,7-\mathrm{C}_{8} \mathrm{H}_{4} \mathrm{Me}_{4}$ (TMCOT) ligands.27.28 We therefore believed that high-quality electronic strueture calculations, with scalar and spin-orbit relativistic corrections, could add significantly to the body of knowledge known about this hard-to-handle molecule.

We recently published a comprehensive paper in which we used gradient-corrected DFT calculations with spin-orbit corrections to calculate the geometry, electronic transitions, ionization energies, and vibrational frequencies of $\mathrm{Pa}(\mathrm{COT})_{2}{ }^{29}$ We showed that the calculated electronic transition energies in the molecule are extremely sensitive to the assumed geometry, especially the $\mathrm{Pa}-\mathrm{COT}$ bond distince. Previous calculations of the optical transitions in the molecule ${ }^{23,24,30}$ (including our own DV$\mathrm{X} \alpha$ calculations) $)^{31}$ were fixed-geometry calculations with a variety of choices for the $\mathrm{Pa}-\mathrm{COT}$ bond length. We therefore fully optimized the geometric structure of $\mathrm{Pa}(\mathrm{COT})_{2}$ under both $\mathrm{D}_{\mathrm{gh}}$ and $\mathrm{D}_{\mathrm{k}}$ point symmetries, and determined that its geometry is intermediate between those of $\mathrm{Th}(\mathrm{COT})_{2}$ and $\mathrm{U}(\mathrm{COT})_{2}$, both of which have been determined crystallographically. ${ }^{32}$ We were then able to calculate the electronic transitions, ionization energies, and vibrational frequencies at the optimized geometry. We determined that, under $D_{8 \mathrm{a}}{ }^{*}$ double-group symmetry, $\mathrm{Pa}(\mathrm{COT})_{2}$ has an $\mathrm{E}_{3 / 20}$ ground state that corresponds to an (f $\left.\phi\right)^{d}$ electron configuration. The lowest-energy electric-dipole-allowed f-d electronic transition in $\mathrm{Pa}(\mathrm{COT})_{2}$ 
is calculated to occur at $368 \mathrm{~nm}$, which is in excellent agreement with the experimental estimation of 365 nm.. ${ }^{28}$ Significantly, our assignment of this band differs from those of earlier calculations, and we believe that our DFT calculations at the optimized geometry provide the assignment that is most consistent with the available experimental data. ${ }^{33}$

In addition to addressing the optical transitions in $\mathrm{Pa}(\mathrm{COT})_{2}$, we also performed comprehensive benchmarking of the calculated vibrational frequencies of the molecule, both with and without the inclusion of spin-orbit coupling. Our calculated vibrational frequenoies and intensities at the scalar relativistic level are in excellent agreement with the available experimental values allowed us to correct some misassignments of the vibrations in the literatures. These studies provided additional support for our notion that theoretical calculation of the vibrations of actinide-containing species can be carried out successfully without the inclusion of spin-orbit effects. Thus, scalar relativistic DFT calculations provide a convenient way to predict quantitatively the vibrational frequencies and intensities of actinide systems that is less computationally demanding than full double-group calculations with spin-orbit coupling.

Recent experimental developments in organoactinide chemistry have stimulated us to explore other aspects of actinide sandwich chemistry. In 1995, Ephritikhine and coworkers reported the serendipitous synthesis of a remarkable anionic uranium sandwich complex with two cycloheptatrienyl ligands. ${ }^{34}$ This complex, $\mathrm{UCh}_{2}{ }^{-}\left(\mathrm{Ch}=\eta^{7}-\mathrm{C}_{7} \mathrm{H}_{7}\right)$, represented an entirely new class of actinide sandwich complex, and the bonding in such systems is expected to provide a nice counterpoint to that in $\mathrm{Ch}$ complexes of the transition elements. ${ }^{33}$ We therefore used gradient-corrected DFT calculations with scalar relativistic corrections to explore the structures, bonding, and relative energetics of a series of neutral and charged actinide-Ch sandwich complexes, $\mathrm{AnCh}_{2}{ }^{9}$ $(\mathrm{An}=\mathrm{Th}, \mathrm{Pa}, \mathrm{U}, \mathrm{Np}, \mathrm{Pu}, \mathrm{Am} ; \mathrm{q}=2-, 1-, 0,1+)^{36}$ There were several notable conclusions to this study. For example, we found that the An f $\delta$ orbitals not only participate in the bonding with the $e_{2}{ }^{n} p \pi$ orbitals of the $\mathrm{C}_{7} \mathrm{H}_{7}$ rings, but are as important as the $\mathrm{An}$ d $\delta$ orbitals in stabilizing the frontier p $\pi$ orbitals of the $\mathrm{C}_{7} \mathrm{H}$, rings. Further, with increasing atomic number of the actinide, the $5 f$ manifold and ligand based frontier MOs become considerably closer in energy. As a result, the actinide $5 f$ percentage in the frontier $\mathrm{e}_{2}$ " MOs increases markedly, while the contribution by the 6 d orbitals gradually decreases. As a consequence, the An$\mathrm{Ch}$ bonding, as gauged by the $\mathrm{An}-\mathrm{Ch}$ bond energy, decreases as one proceeds to the later actinide elements (Figure 1). The implication of this conclusion is that further synthetic explorations of An-Ch chemistry are likely to be more successful with the early actinide elements. Our ability to help chart the synthetic chemistry of actinide complexes has been and we hope continues to be of utility to synthetic chemists in the field. It is also satisfying to note that the correctness of our description of the electronic structure of $\mathrm{UCh}_{2}{ }^{-}$was recently corroborated by an experinental study of the EPR and ENDOR spectra of the anion."

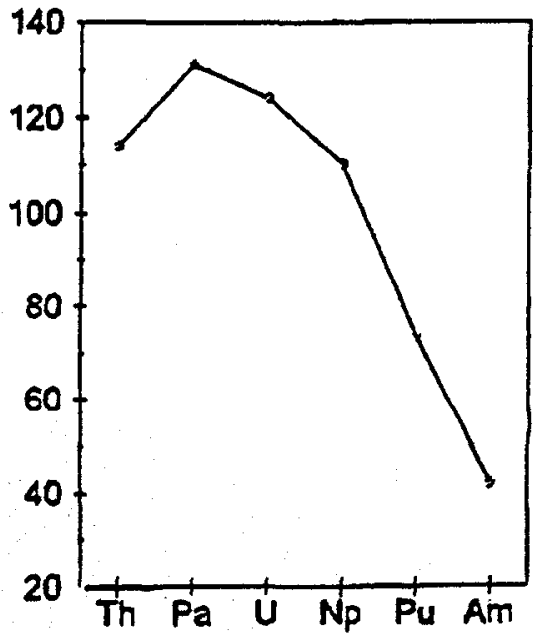

Figure 1. Plot of the homolytic bond energy $\left(D_{c}\right.$ in $\left.\mathrm{kcal} / \mathrm{mol}\right)$ vs. An for the neutral $\mathrm{AnCh}_{2}$ molecules.

We have also begun a comprebensive investigation into actinide sandwich complexes of arenes. Bis(arene) actinide complexes are currently unknown, although there has been evidence for An(arene) ${ }_{2}^{+}$ $\left(\mathrm{An}=\mathrm{Th}, \mathrm{U}\right.$ ) ions in mass spectrometric experiments. ${ }^{38}$ The existence of these ions, in conjunction with an ever-growing chemistry of arenes with lanthanide atoms, has led to several recent investigations into 


\section{C-6}

the electronic structure of $\mathrm{An}\left(\eta^{6}-\mathrm{C}_{6} \mathrm{H}_{6}\right)_{2}$ complexes. ${ }^{39}$ These calculations have invariably assumed a "linear", $D_{6 h}$ structure for the molecules, based largely on the crystallographically determined struoture of $\operatorname{Gd}(\mathrm{TBB})_{2}\left(\mathrm{TBB}=\eta^{6}-1,3,5-\mathrm{C}_{6} \mathrm{H}_{3} \mathrm{BB}_{3}\right)$, which exhibits a linear geometry. ${ }^{10}$ However, in view of the bent structures of several $\mathrm{LnC}_{\mathrm{p}_{2}}$ complexes, ${ }^{41}$ it struck us that the linear structure of $\mathrm{Gd}(\mathrm{TBB})_{2}$ may be largely due to steric interactions rather than an intrinsic eleotronic preferenoe, and that the assumption of a linear structure for $A n\left(\eta^{6}-C_{6} H_{6}\right)_{2}$ complexes might therefore be incorreot. We therefore used relativistic gradient-corrected DFT calculations to calculate the structure of a series of actinide arene sandwich complexes. Our first results, which were recently published, ${ }^{42}$ are summarized in Table 1 and Figure 2.

Table 1 presents the calculated energies for a series of $A n\left(\eta^{6}-1,3,5-C_{6} H_{3} R_{3}\right)_{2}$ as a function of the centroid-An-centroid angle $\theta$. The results show that all of the $A n\left(\eta^{6}-C_{6} H_{6}\right)_{2}$ and $A n\left(\eta^{6}-C_{6} H_{3} M_{3}\right)_{2}$ prefer a bent $\left(\theta<180^{\circ}\right)$ geometry by 4 to $10 \mathrm{kcal} / \mathrm{mol}$. Only when the arene ligands are made extremely bulky by adding three t-butyl groups is the linear $\left(\theta=180^{\circ}\right)$ geomery preferred over the bent one. It is worth noting that the calculations on the $\mathrm{An}\left(\eta^{6}-1,3,5-\mathrm{C}_{6} \mathrm{H}_{3}{ }^{1} \mathrm{Bu}_{3}\right)_{2}$ systems were fully optimized for all 97 atoms

Table 1. DFT (PW91) Energies and Centroid-An-Centroid Angle $\theta$ for An( $\eta^{6}-1,3,5-$ $\left.\mathrm{C}_{6} \mathrm{H}_{3} \mathrm{R}_{9}\right)_{2}(\mathrm{An}=\mathrm{Th}, \mathrm{U}, \mathrm{Pu} ; \mathrm{R}=\mathrm{H}, \mathrm{Me}, \mathrm{Bu})$ Complexes

\begin{tabular}{|c|c|c|c|c|c|}
\hline \multirow[b]{2}{*}{ compound } & \multicolumn{2}{|c|}{ linear } & \multicolumn{2}{|c|}{ bent } & \multirow[b]{2}{*}{$\Delta \mathrm{E}_{\mathrm{E}-\mathrm{L}}(\mathrm{kcal} / \mathrm{mol})^{a}$} \\
\hline & $E(e V)$ & $\theta$ & $E(e V)$ & $\theta$ & \\
\hline $\operatorname{Th}\left(\mathrm{C}_{6} \mathrm{H}_{6}\right)_{2}$ & -156.8969 & $180^{\circ}$ & -157.0940 & $138.5^{\circ}$ & -4.6 \\
\hline $\operatorname{Th}\left(\mathrm{C}_{6} \mathrm{H}_{3} \mathrm{Me}_{3}\right)_{2}$ & -256.4178 & $180^{\circ}$ & -256.6473 & $142.4^{\circ}$ & -5.3 \\
\hline \multirow[t]{2}{*}{$\mathrm{Th}\left(\mathrm{C}_{6} \mathrm{H}_{3} \mathrm{Bu}_{3}\right)_{2}$} & -553.4077 & $180^{\circ}$ & -553.2026 & $170.0^{\circ 6}$ & 4.7 \\
\hline & & & -552.1159 & $160.0^{\circ 6}$ & 29.8 \\
\hline $\mathrm{U}\left(\mathrm{C}_{6} \mathrm{H}_{6}\right)_{2}$ & -157.5073 & $180^{\circ}$ & -157.9475 & $137.0^{\circ}$ & -10.2 \\
\hline$U\left(\mathrm{C}_{6} \mathrm{H}_{3} \mathrm{Me}_{3}\right)_{2}$ & -257.1507 & $180^{\circ}$ & -257.4980 & $139.8^{\circ}$ & -8.0 \\
\hline \multirow[t]{2}{*}{$\mathrm{U}\left(\mathrm{C}_{6} \mathrm{H}_{3} \dot{\mathrm{B}} \mathrm{u}_{3}\right)_{2}$} & -553.8190 & $180^{\circ}$ & -553.5337 & $170.0^{\circ 0}$ & 6.6 \\
\hline & & & -552.1551 & $160.0^{06}$ & 38.4 \\
\hline $\mathrm{Pu}\left(\mathrm{C}_{6} \mathrm{H}_{6}\right)_{2}$ & -156.3889 & $180^{\circ}$ & -156.6706 & $135.3^{\circ}$ & -6.5 \\
\hline $\mathrm{Pu}\left(\mathrm{C}_{6} \mathrm{H}_{3} \mathrm{Me}_{3}\right)$ & -256.0194 & $180^{\circ}$ & -256.2167 & $137.5^{\circ}$ & -4.5 \\
\hline \multirow[t]{2}{*}{$\mathrm{Pu}\left(\mathrm{C}_{6} \mathrm{H}_{3}{ }^{\mathrm{t}} \mathrm{Bu}_{3}\right)_{2}$} & -552.6833 & $180^{\circ}$ & -552.3058 & $170.0^{\circ b}$ & 8.7 \\
\hline & & & -550.6870 & $160.0^{c h}$ & 46.0 \\
\hline
\end{tabular}

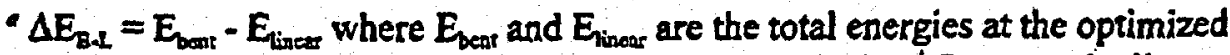
geometries in the bent and linear geometries, respectively. ${ }^{b}$ Because the linear structures are the minima for the $\left(\mathrm{C}_{6} \mathrm{H}_{3} \mathrm{Bu}_{3}\right)_{2} \mathrm{An}$ complexes, the energies for their "bent" structures are calculated at fixed values of $\theta=160^{\circ}$ and $\theta=170^{\circ}$. 
using triple-zetr basis sets and no symmetry constraints. Figure 2 shows the optimized structures of $\mathrm{U}\left(\eta^{6}-1,3,5-\mathrm{C}_{6} \mathrm{H}_{3} \mathrm{Me}_{3}\right)_{2}$ and $\mathrm{U}\left(\eta^{6}-1,3,5-\mathrm{C}_{6} \mathrm{H}_{3} \mathrm{Bu}_{3}\right)_{2}$. This study, which we propose to continue during the next grant period, demonstrates that ligand substituents can have a profound influence on the observed structures of actinide complexes.

The calculations on the $\mathrm{An}\left(\eta^{6}-1,3,5-\mathrm{C}_{6} \mathrm{H}_{3} \mathrm{Bu}_{3}\right)_{2}$ systems, which were carried out at the massively parallelized IBM SP2 supercomputer at the Environmental and Molecular Sciences Laboratory at Pacific Northwest National Laboratory, are among the largest calculations ever carried out on actinidecontaining systems. The geometry optimizations and electronic structure calculations on each An( $\eta^{6}-$ $\left.1,3,5-\mathrm{C}_{6} \mathrm{H}_{3} \mathrm{Bu}_{3}\right)_{2}$ compound require only about 150 - 200 node hours on the IBM SP2 computer, indicating that computational resources are now available to allow us to undertake high-quality DFT calculations on large organoactinide complexes using "real" chemical ligands rather than simplified ligands or pseudo-groups. These sndies further demonstrate the utility of relativistic DFT calculations with respect to very large molecular systems that can't readily be addressed using much more computationally demanding correlated ab initio methodologies. Many of the explorations that we propose below will involve ligand substituent effects on the chemistry of actinide systems, such as in the $\left(C_{s} R_{s}\right)_{2} A n$ and $\left(C_{s} R_{s}\right)_{2} A n X_{2}$ systems.
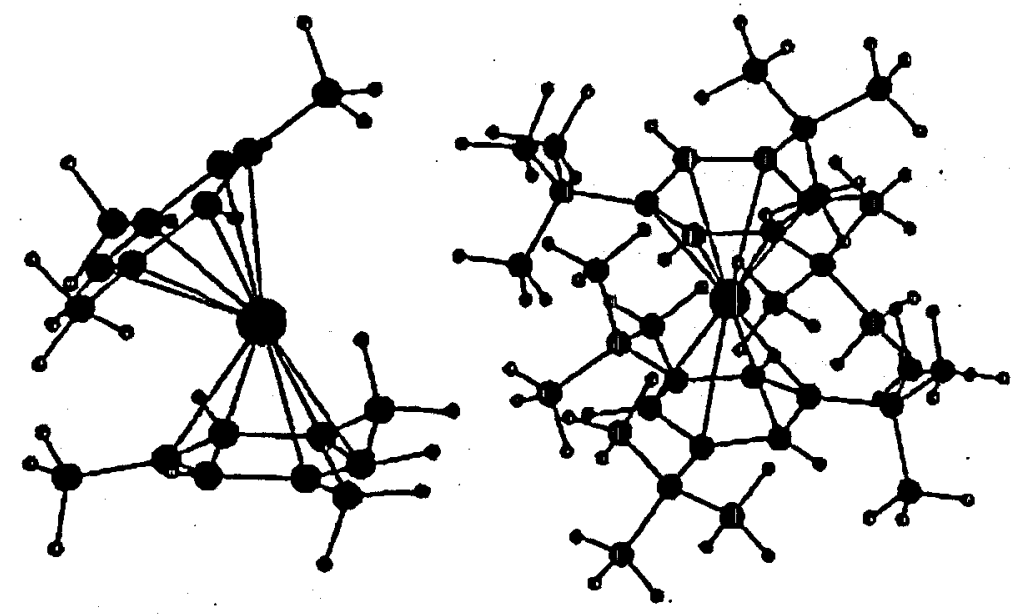

Figure 2. Fully optimized PW91 structures for $\left(\eta^{6}-1,3,5-\mathrm{C}_{6} \mathrm{H}_{3} \mathrm{Me}_{3}\right)_{2} \mathrm{U}$ (left) and $\left(\eta^{6}-1,3,5-\mathrm{C}_{6} \mathrm{H}_{3} \mathrm{Bu}_{3}\right)_{2} \mathrm{U}$ (right). The structures were optimized without imposing specific syrnmetry constraints on the final geometries.

CO Activation by Actinide Atoms. One of the goals of our research program in actinide chemistry is to use electronic structure methods to derive a better understanding of the fundamental interactions between actinide atoms and small molecules. We are also interested in exploring the differences in reactivity and electronic structure as one varies the actinide element. ${ }^{33}$ We have recently begun a collaborative effort with Professor Lester Andrews (University of Virginia) to undertake combined experimental and theoretical research in the reactions of laser-ablated actinide atoms with small molecules, such as $\mathrm{CO}, \mathrm{N}_{2}$, and $\mathrm{CO}_{2}$. The Andrews group is arguably the leading group in the world engaged in the study of the reactions of metal atoms in frozen noble-gas matrices. Our initial efforts have been very successful, as will be discussed briefly here.

We initially explored the rich chemistry that occurs when laser-ablated uranium atoms are cocondensed with $\mathrm{CO}$ in a matrix of solid neon at $4 \mathrm{~K}$. Infrared spectroscopy is the principal probe of the reactions that occur, and isotopic mixtures of $\mathrm{CO}\left({ }^{12} \mathrm{C}^{16} \mathrm{O},{ }^{13} \mathrm{C}^{16} \mathrm{O},{ }^{12} \mathrm{C}^{18} \mathrm{O}\right)$ were used to verify the stoichiometry of various thermal and photochemical reactions. A summary of part of the proposed reaction scheme is given in Scheme 1. We used relativistic DFT calculations to calculate the structures and vibrational frequencies and intensities of all of the proposed species. The agreement between our calculated frequencies and intensities and those observed in the frozen matrices is excellent, which provides strong support for the identification and strucnures of the species. ${ }^{24}$ Because of the accuracy and 


\section{C-8}

the reliability of the calculared DFT frequencies and intensities, our results have strong predictive value for the experimentalists in our collaboration: In certain cases, they had tentatively assigned vibrational bands to a certain species, but our theoretical calculations suggested an alternative assignment that was subsequently found to be superior. We have also been able to predict the band positions of reaction products in advance of the experiments, and these products were later found experimentally. This interplay between experiment and theory has been extremely useful in the discovery of new actinidecontaining molecules in the matrix experiments.

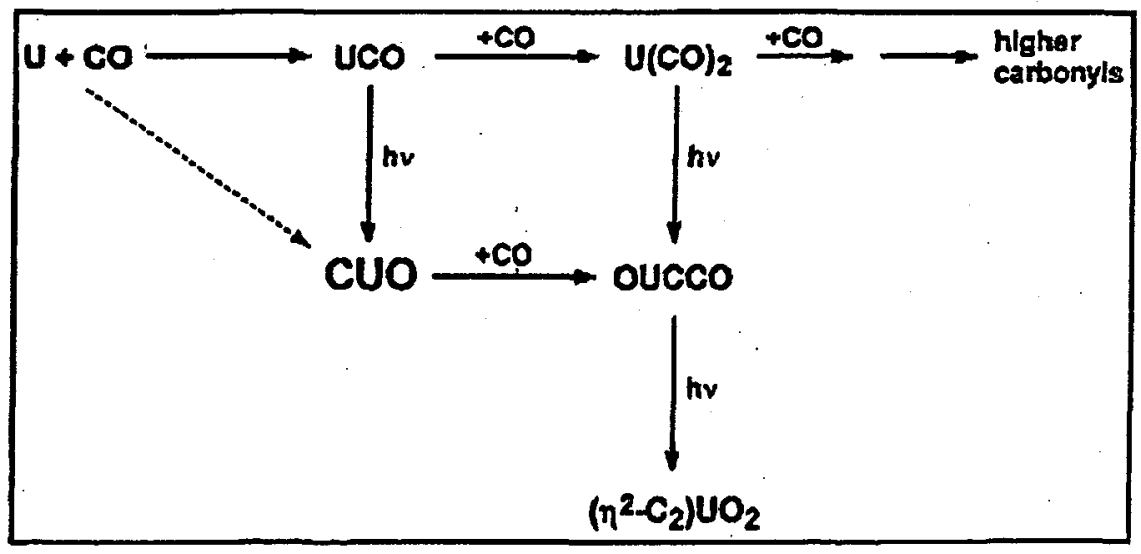

Scheme 1. Summary of reactions that occur between uranium atoms and $\mathrm{CO}$ in frozen Ne.

It is not surprising that the codeposition of $U$ atoms and $C O$ leads to the formation of binary uranium carbonyl complexes, $\mathrm{U}(\mathrm{CO})_{k}$, which will not be discussed in detail here. 'However, the reaction of uranium with $\mathrm{CO}$ also leads to remarkable CO-activation chemistry. For example, one of the prominent species that results from the reaction is the molecule CUO, which is formally a U(VI) complex that is isoelectronic to the ubiquitous uranyl ion, $\mathrm{UO}_{2}{ }^{2+}$. In the course of this reaction, the $\mathrm{U}$ atom is formally inserted into the $\mathrm{CO}$ molecule; the formation of very strong $\mathrm{U}-\mathrm{C}$ and $\mathrm{U}-\mathrm{O}$ multiple bonds makes this reaction exothermic in spite of the cleavage of the very strong C-O triple bond. The experimental and calculared properties of CUO are presented in Table 2. A second CO molecule can react with CUO to form the ketenylidene complex OUCCO. The oxophilicity of $U$ is so great that this molecule

Table 2. Experimental and Calculated (PW91) Spectroscopic and. Structural Data for CUO and CThO

\begin{tabular}{|c|c|c|}
\hline & CUO & CThO \\
\hline$v_{\text {Ano }} \operatorname{expt} /$ calc $\left(\mathrm{cm}^{-1}\right)$ & $872 / 874$ & $812 / 811$ \\
$V_{\text {An }}$ expt $/$ calc $\left(\mathrm{cm}^{-1}\right)$ & $1047 / 1049$ & $618 / 621$ \\
An-O, calc $(\AA)$ & 1.802 & 1.889 \\
An-C, calc $(A)$ & 1.747 & 2.124 \\
$\angle C-A n-O$, calc $\left({ }^{\circ}\right)$ & 180 & 108.9 \\
Ground state, calc & $1 \Sigma^{+}$ & ${ }^{3} \mathrm{~A}^{\prime}$ \\
\hline
\end{tabular}

rearranges to an unprecedented molecule $\left(\eta^{2}-\mathrm{C}_{2}\right) \cup \mathrm{O}_{2}$, which is essentially the adduct of the 
dehyddroacetylene dianion, $\mathrm{C}_{2}{ }^{2}$, to a uranyl ion. The optimized calculated structure of this molecule shows that it contains the first bent uranyl linkage $\left(\angle O-U-O=155.8^{\circ}\right)$, and the correctness of this structure seems to be indicated by the calculated vibrational frequencies and intensities: The calculated antisymmetric and symmetric O-U-O modes for this molecule are 910 and $849 \mathrm{~cm}^{-1}$, respectively, just 12 and $6 \mathrm{~cm}^{-1}$ from the observed values. Both of these modes are calculated to be intense, with the antisymmetric mode correctly predicted to be the stronger.

We have also recently observed that $\mathrm{Th}$ atoms react with $\mathrm{CO}$ to form the first thorium carbonyls and Th-CO insertion products. ${ }^{45}$ One of the insertion products is $\mathrm{CThO}$, which, at first glance, appears to be entirely analogous to CUO. However, the difference in the valence eleotronic structure of $U$ and $T h$ implies that $C T h O$ will exhibit very different bonding from that of CUO. Because Th has only four valence electrons, its maximum oxidation state is +4 , as compared to the +6 oxidation state on $U$ in CUO. We calculate that CThO is a bent molecule $\left(\angle \mathrm{C}\right.$-Th-O $\left.=108.9^{\circ}\right)$ with a triplet ground state (Table 2). The spin

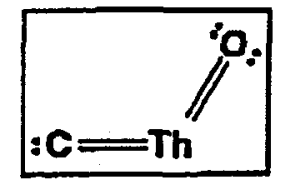
density of the molecule is predominantly localized on the $C$ atom; in essence, CThO is a triplet carbene that corresponds to the simple Lewis structure shown to the right. The fact that we have calculated the correct structure and state of this very unusual molecule seems evident as based on the calculated stretching vibrational frequencies of the molecule, which are both within $3 \mathrm{~cm}^{-1}$ of the experimental values. The Th-C bonding in CThO is much weaker than that in CUO as based on the observed vibrational frequencies and an analysis of the bonding in the molecule. We recently communicated these experimental and theoretical results on $\mathrm{CThO},{ }^{46}$ and we are pleased that these results were of sufficient interest and significance to be highlighted in Chem. Eng. News as a Science Concentrate. We are proposing to continue this research in the next grant period.

Theoretical Studies of Molecules of the Transactinide Elements. One of our goals in the last renewal proposal of this grant was to use theoretical methods to help chart the chemistry of the transactinide elements. Since the time of that proposal, transactinide chemistry has been an extremely vibrant area of research and current events, ${ }^{48}$ in part because of the controversy in the naming of the elements ${ }^{49}$ and because of the recent discoveries of several new transactinide elements. ${ }^{50,51}$ Activity in theoretical studies of the electronic structure of atoms and moleoules of the transactinide elements has increased in parallel with the experimental advances. Because of the high atomic numbers of the transactinide elements, these elements exhibit greater spin-orbit effects than any other family of elements, and the correct calculation of their electronic structures therefore provides a great challenge to current electronic struetural methods. s2 $^{2}$

Our first contribution to transactinide chemistry was a DV-X $\alpha$ study of the bonding in the hypothetical $d^{6}$ earbonyl complex $\mathrm{Sg}(\mathrm{CO})_{6 .}^{30}$. We showed that the bonding in this octahedral complex should be similar to that in $\mathrm{Cr}(\mathrm{CO})_{6}, \mathrm{Mo}(\mathrm{CO})_{6}$, and $\mathrm{W}(\mathrm{CO})_{6}$, and it should differ substantially from that in hypothetical $\mathrm{U}(\mathrm{CO})_{6}$. We have recently completed a more detailed study of octahedral $\mathrm{Sg}(\mathrm{CO})_{6}$, involving complete optimization of the bond lengths, using correlated ab initio methodology." Our principal effort during the present period, however, focused on the later representative elements of the transactinides, particularly elements 114 through 118 . Our goal in these studies was to use theoretical methods to see whether the atoms and compounds of the transactinide elements would fit the patterns of the periodic table. The huge spin-orbit effects exhibited by the elements led to some surprising conclusions to our studies. For example, we found that the spin-orbit splitting between the $7 p_{1 / 2}$ and $7 p_{3 / 2}$ spinors is very large, which has profound effects on the predicted chemistry of these elements. For example, element 114 adopts a closed-shell outer electron configuration of $7 \mathrm{~s}^{2} 7 \mathrm{p}_{12}{ }^{2}$, which is ca. $4 \mathrm{eV}$ lower in energy than its first atomic excited state, which corresponds to a $7 s^{2} 7 p_{1 / 2}{ }^{1} 7 p_{2 / 3}{ }^{1}$ configuration. ${ }^{54}$ The corresponding calculated splitting in $\mathrm{Pb}$, the element above element 114 in Group $4 \mathrm{~A}$, is $<1 \mathrm{eV}$. 
The large spin-orbit effects also have a great effect on the predicted properties of compounds of the transactinide elements. For example, we predict that the transactinide hydrogen halide compound, $\mathrm{H}[117]$, will have an $\mathrm{H}-[117]$ bond that is both unusually long and unusually strong when compared to the trends exhibited by the other hydrogen halides..$^{55}$ In this case, the large splitting of the $7 p$ subshell makes the 8 s orbital energetically accessible for contributing to the bonding. A similar effect is observed in our calculations on the hypothetical transactinide noble-gas tetrafluoride compound, [118] $\mathrm{F}_{4} .6$ Application of the well-known VSEPR model, ${ }^{57}$ which has been very successful for the prediction of the geometries of noble-gas fluorides, ${ }^{38}$ leads to the prediction that [118]F, should be a square-planar molecule analogous to $\mathrm{XeF}_{4}{ }^{\text {s9 }}$. Indeed, if only scalar relativistic effects are included, our relativistic configuration interaction calculations on [118] 4 , predict that the square planar geometry is $\mathrm{ca.} .4 \mathrm{eV}$ lower in energy than the tetrahedral geometry. When the spin-orbit operator is also included, however, the two geometries become nearly equal in energy with the tetrahedral geometry very slightly favored (by $0.24 \mathrm{eV}$ ). It is particularly satisfying to us that these studies on [118] $\mathrm{F}_{\text {a }}$ were published only a few months before the discovery of the element! ${ }^{51}$

In addition to the studies above, we have also initiated a study of the structures of hydrated actinide ions. These studies, which were not proposed in the last grant proposal, will be the basis for part of the proposed research and will be discussed in the section on Proposed Research.

\section{Proposed Researcb}

In this section, we will describe the specific directions in which we plan to continue our research in the electronic structure of heavy element chemistry over the proposed grant period. As noted in the previous section, our efforts in the prior grant periods have placed a heavy emphasis on the transition to newer methodologies, and we feel that this transition to higher level methodologies has been successful. As noted earlier, we antioipate that most of our activities in the proposed grant period will emphasize the application of these methods to interesting chemical problems rather than the development of new methodologies. As will be apparent, many of the problems of interest are natural extensions of our current efforts in heavy element chemistry.

\section{Methodology}

As discussed earlier, we have successfully applied two very different electronic structure methodologies to current problems in heavy-element chemistry: (i) We have used gradient-corrected relativistic density functional theory to study a variety of actinide-containing systems. Gradientcorrected DFT gives us the opportunity to explore the structures and spectroscopic properties of large actinide complexes in addition to facilitating a molecular orbital analysis of the structure and bonding, and (ii) we have used higher-level correlated electronic-structure methods to explore the structures and properties of small molecules of the transactinide elements. Part of our effort during the current grant period has involved examining the validity of gradient-corrected DFT methods as applied to the excited states of some $f^{1}$ systems. From our benchmark calculations on $\mathrm{Pa}_{(\mathrm{C}}\left(\mathrm{C}_{8} \mathrm{H}_{8}\right)_{2}, \mathrm{UF}_{6}$ and $\mathrm{NpF}_{6}$, we have found that by using DFT with the PW91 functional we are able to reproduce the experimental excitation energies with remarkably good accuracy: The average absolute errors over a range of excitation energies turn out to be at least as small or smaller than the computationally much more demanding spin-orbit $\mathrm{Cl}$ or four-component Dirac-Fock CI calculations. These studies suggest that the PW9I method with the inclusion of spin-orbit coupling provides a good means to address the energies of excited states, even for actinide compounds. We will also be exploring the efficacy of newer GGA functionals that have been recently developed..$^{60}$ 


\section{C-11}

During the proposed grant period, we will continue to expand our applications of the gradientcorrected DFT method to actinide systems. We plan to benchmark and implement two major improvements to our current way of doing calculations. First, because actinide systems tend to have a large number of low-lying excited states, they inherently have a large number of low-lying and neardegenerate excited states. Calculation of the multiplet structure of such molecules in the presence of significant spin-orbit coupling, which is one of the most fundamental questions in theoretical actinide chemistry because of its great significance for determining the properties of ground-states and especially the excited-states in DFT formalism, has proven to be a great challenge. This problem has made it difficult to address interesting problems such as the optical transitions in actinide complexes with two or more $5 f$ electrons, such as uranocene. We will be exploring some of the newer methods used to address the multiplet problem in transition metal compounds, ${ }^{61}$ and we believe that these extensions of gradientcorrected DFT methods to many-f-electron systems should prove to be very interesting and promising. Second, we will be testing the implementation of the zeroth order regular approximation (ZORA) approach to the Dirac equation, ${ }^{62}$ which has been implemented in a preliminary form in the most current version of the ADF code. ${ }^{63}$ Application of the ZORA method, which is a two-component approximation to the Dirac equation, is one of the frontier areas in relativistic DFT. The use of the ZORA Hamiltonian overcomes some of the drawbacks of the currently widely-used quasi-relativistic Pauli Hamiltonian, which, in principle, can lead to variational collapse because of its singular behavior at the nucleus. We have encoutered these problems in our own test calculations. Moreover, the ZORA calculations have been able to produce results that are almost indistinguishable from the fully-relativistic four-component Dirac-Fock calculations. ${ }^{64}$ We anticipate a series of interesting results from the caloulations of actinide systems using this ZORA method.

We have also had an active collaboration with the Theoretical Group at PNNL, who is developing the NWChem suite of electronic structure and visualization codes. Our colleagues at PNNL are keenly interested in having us benchmark their codes on large actinide systems, particularly their DFT module, and we are particularly interested in taking advantage of some of the special features of this new code that are applicable to large organoactinide systems. These efforts will continue and increase in the proposed grant period.

We anticipate doing very little further research at Ohio State into the application of correlated electronic struoture methods as applied to transactinide chemistry during the proposed grant period. Most of this research was developed as part of the $\mathrm{Ph}$.D. dissertation of one of the PI's former graduate students, Dr. Clinton Nash. After a productive postdoctoral fellowship at Lawrence Livermore National Laboratory, ${ }^{65}$ Dr. Nash is beginning his independent academic career in Autumn, 1999 as a faculty member at the University of New England. The PI wishes to allow him the freedom to pursue further applications of correlated electronic structure methods to heavy-element systems without "competition" from his Ph.D. advisor!

\section{Specific Chemical Problems}

We will be applying the above methodologies to a number of interesting current problems in actinide chemistry. In most cases, the areas of theoretical investigation have been directly stimulated by questions raised by experimental researchers in the field. Several of the areas that we plan to investigate are detailed below.

Structure, Bonding, and Reaction Dyanmics in Organoactinide Complexes. In the Pr's opinion, our greatest contributions to the area of heavy element chemistry over the previous grant periods have involved the development of generally useful (and often simple) descriptions of the bonding in actinide-containing molecules, particularly actinide organometallics. ${ }^{66}$ These earlier studies were 


\section{C-12}

dictated in part by our need to use quasirelativistic methods, such as the quasirelativistic X $\alpha-S W$ formalism, which could not be used for some of the problems of current interest, such as the correlation of optical spectra. We and several other groups have recently shown that higher level calculations can provide useful quantitative information about the electronic structure and reactivity of felement organometallics. ${ }^{67}$

Our interest in the basic structure and bonding of actinide complexes remains strong and remains a comerstone of this proposal. Because we are now using methods that provide us with meaningful total energies, we have positioned ourselves to be able to determine geometries of complexes, and, more importantly, to begin to address reaction pathways and potential energy surfaces, which are essential aspects of the current interest in the quantum chemistry of inorganic systems. ${ }^{68}$ We will proceed by describing some of the specific organosctinide systems that we have begun investigating and will continue to investigate in the grant period, and for which we have obtained promising preliminary results.

Our recent studies of the actinide sandwich complexes $\operatorname{An}\left(\eta^{n}-C_{n} R_{n}\right)_{2}(n=6,7,8)$ have demonstrated the utility of the GGA DFT methodology for the calculation of large organoactinide molecules and ions. These sandwich systems have the advantage of high, pseudo-axial $D_{n h}$ or $D_{n d}$ symmetry, which makes the calculations more computationally efficient and facilitated the interpretation of the results. We have begun to extend this methodology to lower symmetry systems that are of significant interest in the synthetic organoactinide community.

Much of our initial research in organoactinide chemistry was stimulated by the rich chemistry of cyclopentadienyl complexes of thorium and uranium. We are continuing to investigate interesting new problems in cyclopentadienyl-actinide chemistry. For example, we have initiated a study of the structure and reaction chemistry of the uranium imide complex $\mathrm{Cp}_{2}{ }_{2} \mathrm{U}(\mathrm{NPh})_{2}\left(\mathrm{Cp}^{*}=\eta^{5}-\mathrm{C}_{5} \mathrm{Me}_{5}\right)$, recently synthesized by Burns and coworkers at Los Alamos. ${ }^{69}$ This molecule, which is the first example of a $U(V I)$ organometallic species, is the starting point of a new series of compounds that contain actinide-ligand multiple bonds. The bis(imide) complex has a typical bent metallocene structure with an N-U-N angle of

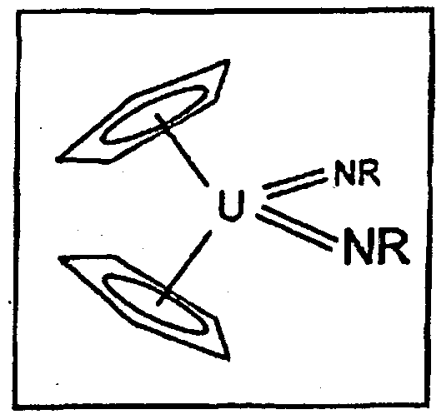
98. $7^{\circ}$. The bent geometry of the formal $[R N=U=N R]^{2+}$ fragment is in stark contrast to the linear geometry invariably observed in derivatives of the isoelectronic uranyl ion, $[\mathrm{O}=\mathrm{U}=0]^{2+},{ }^{70}$ and bears closer resemblance to the bent geometry of $\mathrm{ThO}_{2}{ }^{\prime \prime}$ It is not yet known whether the bent geometry of the $\left[\mathrm{U}(\mathrm{NR})_{2}\right]^{2+}$ is intrinsic to this ion, or whether it is induced, either sterically or electronically, by the $\mathrm{Cp}^{*}$ ligands.

In order to address some of these fundamental questions, we are performing geometry optimizations for a series of $\left[\mathrm{UX}_{2}\right]^{q+}$ and $\mathrm{Cp}_{2} \mathrm{UX}_{2}$ complexes $\left(\mathrm{X}=\mathrm{O}, \mathrm{NH}_{1} \mathrm{CH}_{2}, \mathrm{~N}, \mathrm{CH}, \mathrm{C}, \mathrm{S}, \mathrm{PH}, \mathrm{SiH}, \mathrm{P}\right.$, $\mathrm{SiH}$ and $\mathrm{Si}$ ). Our preliminary calculations show that there is strong $\pi$ bonding between the $d$ and $f$ orbitals of the actinide and the $p$ orbitals of the ligand. In order to assist in advancing the synthesis and reactivity of these systems, we will calculate the relative metal-ligand bond strengths and the vibrational frequencles of some of these species. We will also look at mixed oxa-imido systems, such as $[\mathrm{O}=\mathrm{U}=\mathrm{NH}]^{2+}$, studies that will be relevant to new mixed oxo-imido $\mathrm{U}(\mathrm{VI})$ organometallics, such as $\mathrm{Cp}_{2} \mathrm{U}(\mathrm{O})(\mathrm{NR}) .^{72}$ We will then examine the influence of the $\mathrm{Cp}$ and $\mathrm{Cp} *$ ligands on the observed geometries and on the energies of the 5 felectrons. We believe that these studies will provide us with a much better understanding of the relative influences of multiply bonded ligands, such as oxo and imido, and of organic ligands, such as $\mathrm{Cp}_{\mathrm{p}}$ and $\mathrm{Cp}^{*}$, on the observed geometries of these complexes.

Model studies of the electronic strueture of $\mathrm{Cp}_{2}{ }_{2} \mathrm{U}(\mathrm{NPh})_{2}$ will also afford us the opportunity to 
explore reaction dynamics in organoactinide complexes in terms of total energy. For example, this complex can be synthesized by the direct reaction of 1-lithio-1,2-diphenylhydrazine and $\mathrm{Cp}_{2}{ }_{2} \mathrm{UClMe}$, with concomitant elimination of methane and $\mathrm{LiCl}$. Amey and Burns have postulated an intermediate U(IV) diphenylhydrazido species in the course of this reaction. The $\mathrm{N}-\mathrm{N}$ bond of the diphenylhydrazido ligand presumably undergoes oxidative addition at the U(TV) center, leading to the ultimate U(VI) bis(imido) complex. The relative roles of the $U$ sf and $6 d$ orbitals in such fundamental reactions are unknown. Because of previous limitations in our computational resources, we were unable to perform the multidimensional optimizations necessary to find the experimentally suggested intermediates. By using our newly available computational resources at PNNL, we have found the intermediate of $\mathrm{Cp}_{2}{ }_{2} \mathrm{U}(\mathrm{FN}-\mathrm{NH})$ that has the direct bonding between the $\mathrm{N}$ atoms. We plan to explore this reaction mechanism further, including the use of the actual $\mathrm{Ph}$ substituents on the imide ligands. We are hopeful that we will be able to generalize these result to other reactions at organoactinide centers.

This project will involve close collaboration with experimentalists, particularly those at Los Alamos National Laboratory. In support of these efforts, one of the PI's second-year graduate students is currently a selected participant in the Seaborg Institute Research Fellowship Program for Summer 2000, a special summer program in synthetic and experimental f-element chemistry that is run by the Seaborg Institute at Los Alamos.

Actinide Carbonyl Complexes. We will also continue to extend our calculations on uranium carbonyl complexes, which is one of many classes of novel carbonyl complexes that have been at the forefrom of late. The relative rarity of compounds containing a stable An-CO bond is striking in view of the ubiquity of such bonds among the d-block transition metals. Andersen et al. isolated the first actinide carbonyl complex, $\left(\eta^{3}-\mathrm{C}_{5} \mathrm{H}_{4} \mathrm{SiMe}_{3}\right)_{3} \mathrm{UCO}$, in $1986 .^{14}$ This complex exhibits a CO-stretching frequency of $1969 \mathrm{~cm}^{-1}$, which, being ca. $150 \mathrm{~cm}^{-1}$ lower than that of free CO, indicates significant backdonation to the $\mathrm{CO}$ ligand. We have previously described the bonding in $\mathrm{C}_{3} \mathrm{UCO}$ via quasirelativistic $\mathrm{X} \alpha$-SW calculations, with particular focus on the extent of U Sf - $\mathrm{CO} 2 \pi$ backbonding. ${ }^{75}$

Several other uranium carbonyl complexes have recently been synthesized, including the first crystallographically characterized actinide carbonyl, $\left(\eta^{3}-\mathrm{C}_{3} \mathrm{Me} \mathrm{e}_{4} \mathrm{H}\right)_{3} \mathrm{UCO},{ }^{76}$ and the sterically congested complex $\mathrm{Cp}_{3}{ }_{3}$ UCO. ${ }^{77}$ Table 3 presents some of the available $\mathrm{IR}$ data for the $\mathrm{CO}$-stretching frequencies of these complexes.

Table 3. Experimental CO-stretching Frequencies for $\mathrm{Cp}_{2} \mathrm{UCO}$ Complexes

\begin{tabular}{|l|l|l|}
\hline Cp ligand & $v_{\mathrm{Co}}\left(\mathrm{cm}^{-1}\right)$ & Reference \\
\hline$\eta^{5}-\mathrm{C}_{3} \mathrm{H}_{4} \mathrm{SiMe}_{3}$ & 1969 & 74 \\
$\eta^{3}-\mathrm{C}_{3} \mathrm{H}_{4} \mathrm{CMe}_{3}$ & 1960 & $76(\mathrm{~b})$ \\
$\eta^{5}-\mathrm{C}_{5} \mathrm{H}_{3}\left(1,3-\mathrm{SiMe}_{3}\right)_{2}$ & 1988 & $76(\mathrm{~b})$ \\
$\eta^{5}-\mathrm{C}_{3} \mathrm{Me}_{4} \mathrm{H}$ & 1880 & $76(\mathrm{a})$ \\
$\eta^{5}-\mathrm{C}_{3} \mathrm{Me}_{5}$ & 1905 & 77 \\
\hline
\end{tabular}

The large variation of $v_{c o}$ with the substituents on the $C_{p}$ ring is both striking and nonintuitive. In particular, it is surprising that increasing the number of donor substizuents, in the case of $\mathrm{SiMe}_{3}$ or $\mathrm{Me}$ groups, leads to an increase in $v_{c o}$ rather than the expected decrease. We propose that the steric demands of the various $\mathrm{Cp}$ ligands leads to different degrees of pyramidalization of the $\mathrm{C}_{p_{3}} \mathrm{U}$ fragment and 
different U-Cp(centroid) bond lengths, both of which will affeot the $\pi$-basicity of the fragment. In fact, the crystal structure $\left(\eta^{5}-\mathrm{C}_{3} \mathrm{Me} \mathrm{H}_{4}\right)_{3} \mathrm{UCO}$ indicates that the $\mathrm{Cp}_{\mathrm{p}}$ rings are in a nearly "planar," essentially trigonal planar arrangement, and that the $C p$ rings "gear" nicely with one another. "7 That effect can't be achieved with the larger $\mathrm{Cp}^{*}$ ligands. It is also interesting to note that thermocbemical measurements indicate these uranium carbonyl complexes, albeit exhibiting significant backbonding, are fragile molecules with weak U-CO bonds. ${ }^{73}$ The weak U-CO bonds may be an indication of unusual steric congestion in the $\mathrm{Cp}_{3} \mathrm{UCO}$ complexes.

With the current DFT methodology we are using and the computational resources available to us, we can calculate optimized geometries and vibrational frequencies of all of the complexes listed in Table 3. These studies will allow us to look more closely at several of the above questions, particularly the large influence of the $\mathrm{Cp}$ ligands on the observed $\mathrm{CO}$ stretching frequency, and the apparent kinetic instability of the U-CO bond even in the presence of significant backbonding. Further, they will provide new insight into the basic interactions between $C_{p}$ ligands and actinide elements, information that will be particularly useful in the absence of detailed crystallographic data on these complexes. In addition, an increasing number of catalytic processes involving f-element and transition metal organometallics have been shown to depend critically on the steric demands of ligands such as $\mathrm{Cp} .79$ We believe that these calculations will provide important benchmarks for the use of advanced DFT methods to explore substituent effects on the structures of organoactinide and organotransition metal complexes.

Small Matrix-Isolated Actinide-Containing Species. As mentioned in the Progeress to Date section, we have established a very fruitful collaboration with the experimental group led by Professor Lester Andrews at the University of Virginia. Our collaborative efforts have charted some fundamental reactions of actinide atoms with small molecules, such as $\mathrm{CO}$ and $\mathrm{CO}_{2}$, and has given us the opportunity for detailed studies of the structure and bonding in small actinide-containing species. We propose to continue this collaboration during this grant period.

As noted earlier, our collaborative efforts with the Andrews group has helped establish the strucures of the adducts of $U$ and $\mathrm{Th}$ atoms with $\mathrm{CO}$ in neon matrices. These reactions are complex, and recent studies have shown the importance of defining good potential energy surfaces and state energies for the molecules. As we reported earlier, the reaction of $U$ with $\mathrm{CO}$ in neon leads to the formation of CUO among other products. ${ }^{4}$ The isolated CUO molecule is predicted to have a closed-shell ground state and a linear geometry, just as is observed for the uranyl ion, $\mathrm{UO}_{2}{ }^{2+}$, with which it is isoelectronic. The vibrational frequencies and intensities that are predicted from relativistic DFT calculations match those observed in the neon matrix remarkably well. Surprisingly, when the same experiment is done with a host of argon atoms, a very different vibrational spectrum emerges for CUO. ${ }^{\text {BO }}$ Our calculations lead to the prediction that the electronic state of CUO in solid Ar corresponds to an open-shell excited state of the isolated CUO molecule. This excited state, which lies less than $2 \mathrm{kcal} / \mathrm{mol}$ above the ground state, corresponds to the transfer of a C-based electron to an empty $U$ Sf orbital, thus leading to an $f^{\prime}$ complex. This excited state lies much closer to the ground state in $\mathrm{CUO}$ than it does in $\mathrm{UO}_{2}{ }^{2+}$ because of the lower electronegativity of carbon as compared to oxygen.

Our preliminary exploration of the manifold of excired states of CUO (and, by extension, CThO) reveals it to be far more complicated than we first thought. Further, these studies reveal shortcomings in the DFT method because of difficulties in correctly describing closely-spaced degenerate states. We plan to explore these molecules in greater detail by using Bartlett's coupled-cluster method with singles, . doubles, and noniterative miples [CCSD(T)] method," which provides among the highest quality correlated calculations. We have successfully used Bartlett's ACES II software" in our studies of small molecules of the transactinide elements, and we are optimistic that it will provide a good description of the states of the small actinide adducts. We also plan to use CASSCF, MRCI and spin-orbit CI methods 
to explore the potential energy surfaces of some of the unique small actinide new molecules, such as $\mathrm{NUN}, \mathrm{UO}_{2}$, and $\mathrm{NThO}$, to add insight into the reaction mechanism for the formation of these small molecules.

The Andrews group has started parallel explorations of the reactions of actinide atoms with other small molecules, such as $\mathrm{CO}_{2}, \mathrm{~N}_{2}, \mathrm{O}_{2}, \mathrm{NO}$, and alkanes. The results they have obtained thus far are quite significant. For example, $U$ atoms react with $\mathrm{CO}_{2}$ to form the oxo-carbonyl complex OUCO, which is the first example of a U(II) complex. Our initial DFT calculations on this molecule again lead to excellent agreement with the experimental results. ${ }^{22}$ We will continue these studies, including explorations of the reactions of Th with $\mathrm{CO}_{2}$, and reactions that involve $\mathrm{O}_{2}$ and $\mathrm{NO}$ as the reactive substrates in the noble-gas matrix. We have also started simulation studies of the effects of the noble-gas matrix via calculations on clusters such as CUO $\left(\mathrm{Ng}_{\mathrm{a}}(\mathrm{Ng}=\mathrm{Ne}, \mathrm{Ar} ; \mathrm{n}=13,17,20,23)\right.$. We are hopeful that these theoretical studies, combined with the appropriate experiments, will greatly increase the basic notions of bonding for the actinide elements.

Applications of GGA DFT Methods to Other f' Systems. As reported above, we have been very successful in the application of the PW91 method to optical transitions in $f^{\prime}$ systems. We are proposing to extend this approach to other $f^{\prime}$ systems to verify further the effectiveness of our computational approach and to provide further comparisons to SOCI or DFCI calculations. The systems to be studied will include the $\mathrm{M}\left(\mathrm{C}_{8} \mathrm{H}_{8}\right)_{2} \cdot(\mathrm{M}=\mathrm{Th}, \mathrm{Ce}) \mathrm{COT}$ sandwich complexes and the recently characterized $\mathrm{Th}$ (pentalene) ${ }_{2}$ anion. ${ }^{\mathbf{3}}$ Our preliminary calculations for the $\mathrm{Th}\left(\mathrm{C}_{8} \mathrm{H}_{\mathrm{B}}\right)_{2}{ }^{\circ}$ system has already found good agreement to the recently reported experimental UV-vis spectrum of this compound. ${ }^{\text {s4 }}$

In addition to addressing the optical spectra of these complexes, these calculations will allow us to calculate and extend our understanding of the magnetic properties, ESR g-factors and molecular hyperfine interactions, which can now be calculated using the ZORA approach. ${ }^{62}$ We have begun benchmark ZORA calculations on the UF $(n=1-6)$ and $\mathrm{AnF}_{6}$ systems to test the success of the method in calculating the ground state geometries, vibrational frequencies, and excited state energies of actinide systems. Since this is a very new method, we will benchmark the calculations to explore the advantages and the drawbacks of this method to the actinide systems. The magnetic properties of these systems will also be addressed. Our preliminary calculations indicate good agreement with the experimentally measured values. The extension of this methodology to the optical transitions of $\mathrm{f}^{\mathbf{l}}$ systems will also provide comparisons between the $4 \mathrm{f}$ and $5 \mathrm{f}$ systems and will help us to explain the unique nature of $\mathrm{Th}$ in its trivalent state.

Optical Transitions in $\mathbf{f}$ Complexes. One of the greatest and most relevant challenges in theoretical actinide chemistry is the calculation of optical $f$ - $f$ transitions in molecules that contain an actinide center with more than one metal-localized electrons. For example, the extensive coordination and organometallic chemistry developed for $U(T V)\left(f^{2}\right), U(I I I)(f), P u(V I)(f), P u(V)(f)$, and $P u(I I I)\left(f^{f}\right)$ has generated a wealth of electronic spectral data that have not been fully interpreted in light of electronic structure calculations. Determining the relative state energies of these complex electronic systems is complicated by the problem of multiplets that are subject to significant spin-orbit effects. Because of these complications, we have focused on $f^{\prime}$ complexes [such as $\mathrm{Pa}\left(\eta^{2}-\mathrm{C}_{2} \mathrm{H}_{6}\right)_{2}$ ] in our calculations of electronic transitions. During the next grant period, we believe that we will make significant progress in the first-principles calculation of the electronic transitions of $f$ complexes.

We plan to extend the current Ziegler-Dahl multiplet method to include spin-orbit coupling." The recent applications of CIS-DFT or CAS-DFT methods to multiplet problems have paved the way for further extension of these methods to including spin-orbit effects. As an alternative means of approaching the multiplet problem, we will also look at the inclusion of spin-orbit effects in the time. 


\section{C-16}

dependent-DFT methods, which has recently been found to be a promising method for calculating excitation energies and multiple energies. ${ }^{26}$ We are hopeful that these theoretical constructs will allow us to calculate the state energies of many-f-electron systems with the simultaneous inclusion of spin-orbit effects. We will initially apply these methods to a number of important $f$ systems, such as $U X_{4}(X=$ talide, alkoxide), $\mathrm{U}\left(\eta^{8}-\mathrm{C}_{8} \mathrm{H}_{8}\right)_{2}$, and $\left[\mathrm{PuO}_{2}\left(\mathrm{H}_{2} \mathrm{O}\right)_{5}\right]^{2 *}$.

Theoretical Studies of Hydrated Actinide Ions. A current priority of the DOE is the remediation of the nuclear waste generated by the processing of spent nuclear fuel over the last SO+ years. $^{87}$ The majority of this waste is in the form of ill-defined aqueous sludges that exhibit complex chemistry that is highly dependent on $\mathrm{pH}$. There have been an increasing number of recent experimental studies of the speciation of actinides, ${ }^{88}$ particularly plutonium, ${ }^{89}$ which add to our understanding of their environmental chemistry and how they can better be detected. Structural characterization of actinidecontaining species in sludges and brines are extremely difficult, however, and it is an area in which theoretical structure calculations can assist the experimentalists. In collaboration with theoretical and experimental colleagues at Argonne National Laboratory, we have recently expanded our studies of actinide complexes to include calculations on model hydrated complexes of actinide and actinyl ions.

We recently published our first contribution in this area, namely a relativistic DFT study of the structures of hydrated $\mathrm{Pu}^{3+}$ ions, $\mathrm{Pu}\left(\mathrm{H}_{2} \mathrm{O}\right)_{n}{ }^{3+}(\mathrm{n}=6,8,9,10) .^{90}$ These calculations predicted a preferred first-solvation-shell coordination number of eight or nine and $\mathrm{Pu}-\mathrm{O}$ bond lengths of 2.51-2.55 $\AA$. The calculated bond length is in excellent accord with the available EXAFS data for $\mathrm{Pu}^{3+}$, and the calculated coordination number is in reasonable agreement with two recent analyses of the EXAFS data (which do not agree with one another!). ${ }^{91}$ Our collaboration with the Argonne group has extended to a recent sudy of neptunium ions in water, which was recently submitted for publication. ${ }^{22}$

These DFT studies on hydrated actinide ions have been sufficiently promising that we propose extending these studies to the hydration of other $\mathrm{An}^{q^{+}}$and $\mathrm{AnO}_{2}{ }^{q+}$ jons. We have begun calculations on the hydrated actinyl ions, $\mathrm{AnO}_{2}{ }^{2+}(\mathrm{An}=\mathrm{U}, \mathrm{Np}, \mathrm{Pu})$, in order to explore the structures of and bonding in these actinide speciation complexes. Similar DFT studies have also recently been initiated by Hay, Martin, and Schreckenbach at LANL." The theoretical advances in DFT, including Ziegler's implementation of methodology for the conductor-like screening model (COSMO), enables us to explicitly include solvent effects and to optimize the structures and calculate vibrational frequencies of the solvated ions. The COSMO calculations should allow for effective theoretical modeling of the redox properties of the actinide ions, which is an important part of their chemistry in waste sites. We anticipate that these studies will provide fundamentally interesting results that are highly relevant to experimental studies in progress at the national laboratories. 